\title{
Magnetization Transfer Imaging in Premanifest and Manifest Huntington Disease: A 2-Year Follow-Up
}

\author{
S.J.A. van den Bogaard, E.M. Dumas, E.P. Hart, J. Milles, R. Reilmann, J.C. Stout, D. Craufurd, C.R. Gibbard, S.J. Tabrizi,
} M.A. van Buchem, J. van der Grond, and R.A.C. Roos

\begin{abstract}
BACKGROUND AND PURPOSE: MTI is a quantitative MR imaging technique that has recently demonstrated structural integrity differences between controls and patients with HD. Potentially, MTI can be used as a biomarker for monitoring disease progression. To establish the value of $\mathrm{MTI}$ as a biomarker, we aimed to examine the change in these measures during the course of HD.
\end{abstract}

MATERIALS AND METHODS: From the Leiden TRACK-HD study, 25 controls, 21 premanifest gene carriers, and 21 patients with manifest HD participated at baseline and during a 2-year follow-up visit. Brain segmentation of the cortical gray matter, white matter, caudate nucleus, putamen, pallidum, thalamus, amygdala, and hippocampus was performed by using the automated tools FAST and FIRST in FSL. Individual MTR values were calculated from these regions, and MTR histograms were constructed.

RESULTS: In the premanifest HD group stage "far from disease onset," a significant increase in MTR peak height of the putamen was observed with time. During the manifest HD stage, neither the mean MTR nor the MTR peak height showed a significant change during a 2-year follow-up.

CONCLUSIONS: MTI-derived measures are not suitable for monitoring in Huntington disease during a 2-year period because there was no decrease in structural integrity detected in any of the manifest HD groups longitudinally. The finding of increased putaminal MTR peak height in the premanifest far from disease onset group could relate to a predegenerative process, compensatory mechanisms, or aberrant development but should be interpreted with caution until future studies confirm this finding.

ABBREVIATIONS: $\mathrm{HD}=$ Huntington disease; $\mathrm{HD} 1=$ manifest $\mathrm{HD}$ stage $1 ; \mathrm{HD} 2=$ manifest $\mathrm{HD}$ stage $2 ; \mathrm{MTI}=$ magnetization transfer imaging; $\mathrm{MTR}=$ magnetizationtransfer ratio

M TI is an MR imaging technique that has been developed to perform structural imaging in a different, possibly more sensitive, way because it has demonstrated abnormalities in the normal-appearing gray and white matter on conventional MR imaging in MS research. ${ }^{1,2}$ The technique of MTI is based on the

Received February 29, 2012; accepted after revision April 27.

From the Department of Neurology (S.J.A.v.d.B., E.M.D., E.P.H., R.A.C.R.), Division of Image Processing, Department of Radiology (I.M.), and Department of Radiology (M.A.v.B., J.v.d.G.), Leiden University Medical Center, Leiden, the Netherlands; Department of Neurology (R.R.), University of Munster, Munster, Germany; School of Psychology and Psychiatry (J.C.S.), Monash University, Victoria, Australia; Manchester Academic Health Sciences Centre and Central Manchester University Hospitals NHS Foundation Trust (D.C.), University of Manchester, Manchester, UK; and Department of Neurology (C.R.G., S.J.T.), Institute of Neurology, University College London, London, UK.

This work was supported by the CHDI/High Q Foundation, a not-for-profit organization.

Please address correspondence to S.J.A. van den Bogaard, MD, Department of Neurology, Leiden University, Rapenburg 70, 2311 EZ Leiden, the Netherlands; e-mail: s.j.a.van_den_bogaard@lumc.nl

Indicates article with supplemental on-line appendix and tables.

http://dx.doi.org/10.3174/ajnr.A3303 exchange of 2 pools of protons: 1 bound to macromolecular structures and 1 bound to free water molecules. ${ }^{3}$ MTI has first and foremost been applied in patients with MS; however, many other diseases have been studied by using MTI, such as Alzheimer disease, ${ }^{4}$ Parkinson disease, ${ }^{5}$ and HD. ${ }^{6,7}$

Previous MR imaging studies in HD mainly focused on the search for a biomarker for monitoring disease progression. In this field lies a great opportunity to study the potential of MTI at very early stages of neurodegeneration. The genetic defect that is responsible for HD is located on the short arm of chromosome 4 and has an autosomal dominant inheritance pattern with full penetrance. ${ }^{8}$ Therefore, when the gene defect has been established in an individual, this inevitably leads to a certain clinical diagnosis at some point in his or her life. Mean disease onset is approximately 35-40 years of age, with a wide range partly depending on the length of the abnormal cytosine/adenosine-guanine repeat. ${ }^{9}$ The ability to establish the gene defect well before any symptoms are present gives a unique opportunity to study asymptomatic gene carriers, commonly referred to as "premanifest gene carriers." 
In previous structural MR imaging studies, significant brain disturbances in terms of striatal atrophy, ${ }^{10,11}$ white matter disturbances, ${ }^{12,13}$ cortical thickness reductions, ${ }^{14}$ and whole-brain volume reduction ${ }^{10,15}$ have been described. These studies all used either conventional MR imaging or DTI. Some of these measures are well-established; however, no criterion standard for disease monitoring by MR imaging currently exists. In essence, what technique and what region are most suitable as a biomarker? Possible problems in HD are nonlinear and nonuniformly affected brain regions. The need for further research for a robust and sensitive MR imaging measure to monitor disease onset and progression is still evident.

Previous work in HD by using MTI consists of 4 reports, all cross-sectional in design. ${ }^{6,7,16,17}$ The main outcome measures used were mean MTR and MTR peak height and are thought to represent structural integrity. ${ }^{17-19}$ Mean MTR represents the average MTR value of all voxels in a region of interest, with a lower mean MTR corresponding to a loss of tissue integrity. MTR peak height reflects the most frequently occurring MTR value in a region of interest when all the MTR values are set out in an MTR histogram, again with lower peak height being associated with loss of structural integrity. Results from these previous studies show reduced structural integrity in patients with early-manifest HD compared with healthy controls or in premanifest gene carriers in multiple regions, namely the white matter, cortical gray matter, caudate nucleus, putamen, pallidum, thalamus, and amygdala. The results are suggestive of the potential of MTI as a biomarker. ${ }^{6,7}$ However, no longitudinal reports are available, to our knowledge.

TRACK-HD is specifically designed to determine the most valuable measures to monitor disease progression. ${ }^{20}$ This study followed both premanifest gene carriers and patients with earlymanifest HD for several years. To determine the true value of any potential (MR imaging) biomarker, longitudinal confirmation is crucial. Therefore, we aimed to examine whether MTI measures change during a 2-year period during the progressive course of HD. Second, if any longitudinal change in a group was present, we sought to determine the correlation to clinical measures of disease progression.

\section{MATERIALS AND METHODS \\ Subjects}

Participants were recruited from the Leiden site of the TRACK-HD study. From the baseline cohort $(n=90)$ in 2008, 78 participants could be included. This cohort consisted of 3 groups: 28 healthy controls, 25 premanifest gene carriers, and 25 patients with early-manifest HD. Inclusion criteria for the premanifest genecarrierconsisted ofageneticallyconfirmed expanded cytosine/ adenosine-guanine repeat $\geq 40$, a disease burden score (calculated as: [(Cytosine/Adenosine-Guanine Repeat Length 35.5) $\times$ Age] of $>250,{ }^{21}$ and the absence of motor abnormalities on the Unified Huntington's Disease Rating Scale, defined as a Total Motor Score of $\leq 5$. Inclusion criteria for patients with early-manifest HD consisted of a genetically confirmed cytosine/ adenosine-guanine repeat $\geq 40$ and the presence of motor abnormalities on the Unified Huntington's Disease Rating Scale-Total Motor Score of $>5$. Also a Total Functional Capacity of 7 or higher was required to ensure that the patients in the early-manifest HD group were in the early disease stage. Subdivision of the premanifest group was made on the basis of their expected years to onset, calculated by the formula from Langbehn et al. ${ }^{9}$ This results in a premanifest HD group "far from expected disease onset" and a premanifest HD group "close to expected disease onset."

Subdivision of the manifest group was made by their staging according to the Shoulson and Fahn Scale, based on the Total Functional Capacity score, resulting in HD 1 and HD 2. HD 1 describes a group of manifest HD in the earliest stage after disease onset, with only minor symptoms. HD 2 is the next stage in the disease with increased symptoms and impact on daily activities, but these patients are still considered early manifest. Healthy gene-negative family members, spouses, or partners were recruited as control subjects. Exclusion criteria consisted of significant (neurologic) comorbidity, active major psychiatric disturbance, and MR imaging incompatibility. Full details on recruitment are available in the TRACK-HD baseline article. ${ }^{10}$ Local institutional review board approval and written informed consent were obtained from all participants.

The same cohort was scanned 24 months later within a 6-week window of their follow-up date. Of the 78 participants, 67 were available for follow-up scanning in 2010 with the MTI protocol included. Reasons for unavailability included too advanced disease stage, time restraints on the full TRACK-HD scanning protocol, and unspecified reasons for withdrawal.

\section{Imaging Sequences}

At both time points, exactly the same study protocol was enforced. The scanning protocol described in our cross-sectional report $^{6}$ was performed in an identical manner 2 years after the baseline visit. In short, all participants underwent scanning on a 3T whole-body scanner (Philips, Best, the Netherlands) with an 8 -channel receive and transmit coil. 3D T1-weighted sequences $\left(\mathrm{TR}=7.7 \mathrm{~ms}, \mathrm{TE}=3.5 \mathrm{~ms}\right.$, flip angle $=8^{\circ}$, matrix size $=224 \times$ $224 \times 164 \mathrm{~mm}$, voxel size $=1.0 \times 1.0 \times 1.0 \mathrm{~mm}$, acquisition time $\sim 9$ minutes) and $3 \mathrm{D}$ gradient MTI sequences (TR $=100 \mathrm{~ms}$, $\mathrm{TE}=11 \mathrm{~ms}$, flip angle $=9^{\circ}$, matrix $=224 \times 180 \times 144 \mathrm{~mm}$, voxel size $=1.0 \times 1.0 \times 7.2 \mathrm{~mm}$, acquisition time $\sim 3$ minutes) were acquired. For the MTI sequences, 2 consecutive imaging sets were acquired, 1 with and 1 without a saturation pulse. A sinc pulse of $25 \mathrm{~ms}$ with a maximal B1 of $10 \mathrm{uT}$ and 2 sidelobes on an off-resonance frequency of $1100 \mathrm{~Hz}$ was applied. Total scanning time for the T1WI and MTI sequences was maximally 12 minutes.

\section{Postprocessing}

The postprocessing pipeline was identical to that described previously in the cross-sectional result article. ${ }^{6}$ T1-weighted images were segmented by using the FAST ${ }^{22}$ and FIRST ${ }^{23}$ tools from the fMRI of the Brain Software Library (http://www.fmrib.ox.ac.uk/ fsl/). ${ }^{24}$ This provided individual brain masks for the following: total white matter, cortical gray matter, caudate nucleus, putamen, pallidum, thalamus, amygdala, hippocampus, and whole brain. To correct for possible partial volume effects, we created an eroded mask of these segmentations by removing 1 voxel in-plane 
Group characteristics for controls, premanifest HD groups A + B, and manifest HD groups 1 and $2^{\mathrm{a}}$

\begin{tabular}{|c|c|c|c|c|c|c|}
\hline & & Controls & Premanifest A & Premanifest B & Manifest 1 & Manifest 2 \\
\hline No. & & 25 & 10 & 11 & 9 & 12 \\
\hline Age & Baseline & $48.3(7.6)$ & $45.5(5.2)$ & 42.9 (11.2) & 47.7 (11.8) & $50.9(9.4)$ \\
\hline CAG & Baseline & Baseline & $41.3(1.3)$ & $44.0(3.1)^{\prime}$ & $43.8(3.5)$ & $43.2(1.9)$ \\
\hline YTO & Baseline & Baseline & $13.8(3.8)$ & $8.4(1.6)$ & $\mathrm{N} / \mathrm{A}$ & $\mathrm{N} / \mathrm{A}$ \\
\hline \multirow[t]{2}{*}{ TMS } & Baseline & Baseline & $2.3(1.7)$ & $3.0(1.1)$ & $16.9(8.8)$ & 26.2 (11.7) \\
\hline & Follow-up & $1.7(1.4)$ & $6.0(6.9)$ & $6.3(2.5)^{b}$ & $23.0(9.0)^{b}$ & $37.8(14.3)^{b}$ \\
\hline \multirow[t]{2}{*}{ TFC } & Baseline & $12.9(0.2)$ & $12.7(0.7)$ & $12.6(0.9)$ & $12.0(1.0)$ & $8.6(1.2)$ \\
\hline & Follow-up & 12.81 & $12.5(0.9)$ & $12.4(1.2)$ & $11.2(2.0)$ & $6.0(3.3)^{\mathrm{b}}$ \\
\hline \multirow[t]{2}{*}{ MMSE } & Baseline & $29.2(1.2)$ & $29.0(1.2)$ & $28.4(1.9)$ & $29.0(0.9)$ & $26.5(3.1)$ \\
\hline & Follow-up & $29.1(1.2)$ & $29.3(0.8)$ & $28.6(1.7)$ & $28.7(1.1)$ & $27.0(3.9)$ \\
\hline \multirow[t]{2}{*}{ SDMT } & Baseline & $50.1(9.6)$ & $52.7(7.4)$ & $46.9(12.0)$ & $42.0(8.1)$ & 31.2 (11.1) \\
\hline & Follow-up & $51.5(10.8)$ & $52.7(9.5)$ & $48.3(9.9)$ & $39.6(10.7)$ & $27.9(13.3)$ \\
\hline \multirow[t]{2}{*}{ Stroop } & Baseline & 99.8 (13.4) & $97.1(10.5)$ & $86.9(16.3)$ & 88.4 (13.6) & $69.4(21.5)$ \\
\hline & Follow-up & $102.7(16.9)$ & $92.5(7.5)$ & $83.4(19.3)$ & 89.8 (18.9) & $55.6(22.6)^{b}$ \\
\hline \multirow[t]{2}{*}{ PBA } & Baseline & $6.4(8.4)$ & $6.7(8.7)$ & $7.4(7.1)$ & 11.7 (11.7) & $14.0(11.8)$ \\
\hline & Follow-up & $6.6(9.0)$ & $6.1(11.9)$ & $7.8(9.70$ & 9.7 (11.7) & $22.6(16.3)$ \\
\hline
\end{tabular}

Note:-CAG indicates cytosine-adenosine-guanine-repeat length; YTO, expected years to disease onset; TMS, total motor score; TFC, total functional capacity; MMSE, Mini-Mental State Examination; SDMT, Symbol-Digit Modality Test; PBA, Problem Behavior Assessment; N/A = not applicable.

${ }^{a}$ Values are mean (SD).

${ }^{\mathrm{b}} \mathrm{P}<.005$ significant longitudinal change.

for all the above-named VOIs. All brain masks were then registered to the MTI volumes by using the transform obtained from linear registration of the T1WI volume with $7 d f$ (FSL FLIRT). MTR was calculated per voxel as M0 - Ms/M0, where Ms is the saturated image, and M0, the unsaturated image. The mean MTR per VOI was calculated. Additionally, to represent the variations of voxel-based MTR within each VOI, we constructed MTR histograms. The MTR peak height was normalized for the size of the volume of interest. MTR peak height and mean MTR were the primary outcome variables. For correction of the (largely unknown) influence of age on MTI values, the MTR parameters were calibrated to the control values, assuming no changes in the control subjects. This procedure entailed scaling the individual premanifest and manifest values according to the mean MTR parameter difference between baseline and follow-up of the control group, which could either be an increase or a decrease with time. In On-line Table 5, actual values of the control group on both time points are available.

To compare the biomarker potential of MTI with volumetric analysis, we calculated volumes for all subcortical regions, white matter volume, and whole brain volume by using the FSL tools FIRST $^{23}$ and SIENAX. ${ }^{25}$ The volume calculation and a correction for intracranial volume were performed as described previously. ${ }^{11}$

\section{Clinical Measures}

A total measure of motor dysfunction was obtained with the Unified Huntington's Disease Rating Scale-Total Motor Score (range, 0-124). Total Functional Capacity score (range, 0-13) and Mini-Mental State Examination score for global assessment of cognitive functioning (range, 0-30) were obtained. Cognitive scores included the total scores from the Symbol Digit Technique Test and the Stroop word-reading card. For assessment of psychiatric disturbances, the Problem Behavior Assessment-short version was used. For a more detailed description of these clinical assessments, see Tabrizi et al (2009). ${ }^{10}$

\section{Statistics}

To examine the longitudinal change in clinical measures, we used MTI and volumetric values (corrected for intracranial volumes) per group; paired $t$ testing per group was used. $\alpha$ was set to .05 to be significant. Second, to account for multiple testing (15 regions), we applied a Bonferroni correction, resulting in an $\alpha<$ .0033 for the strongest findings. For correlation analysis between significant longitudinal findings, we applied a Pearson correlation to the difference between the 2 time points of the clinical and MTI values.

\section{RESULTS}

Group characteristics and clinical measures on both the baseline visit and the follow-up visit are shown in Table. A significant longitudinal increase in motor disturbances is evident in premanifest close to disease onset, HD 1, and HD 2. Global functioning as measured by the Total Functional Capacity significantly decreased in 2 years in the HD 2 group as did the cognitive measure of Stroop word-reading.

All MTI values for 15 VOIs are displayed in On-line Table 1 for the premanifest HD groups and in On-line Table 2 for the manifest HD groups. In premanifest far from disease onset, 5 subcortical gray matter regions showed an increase in MTI parameters, namely the mean MTR of the right caudate nucleus $(P=.049)$, MTR peak height of the right putamen $(P=.003)$, MTR peak height of the left pallidum $(P=.032)$, mean MTR of the right thalamus $(P=.047)$, and MTR peak height of the right amygdala $(P=.050)$. However, after Bonferroni correction, the only statistically significant variation was the MTR peak height of the right putamen. In the premanifest close to disease onset group, the cortical gray matter showed a longitudinal reduction in mean $\operatorname{MTR}(P=.020)$, and the left hippocampus, an increase in MTR histogram peak height $(P=.037)$. For the HD 1 group, the right amygdala mean MTR decreased $(P=.036)$ in the 2-year follow-up period. Only the left amygdala mean MTR showed a reduction $(P=.022)$ in the HD 2 group. None of the results in the premanifest close to disease onset, HD 1, or HD 2 groups 
were statistically significant after correction for multiple comparisons.

The statistical analysis procedure was also applied to a 3-group division instead of the 5-group division, encompassing the total premanifest group (Pre-HD A + B) and the total manifest group $(\mathrm{HD} 1+2)$ and the control group. This was performed to increase the power of the study; however, in essence no additional information could be gathered from this analysis. The On-line Appendix contains exact details of the findings. The results also display subcortical MTR parameter increases in the premanifest group and a decrease in MTR parameters of the amygdala in the manifest HD group. The subdivision of groups is, therefore, more informative because it gives more exact disease stage-related changes.

Correlation analysis of the significant longitudinal findings (without correction for multiple comparison) in MTI values with clinical measures resulted in 2 significant findings-namely, in the premanifest close to disease onset group, the MTR peak height of the left hippocampus correlated to Total Functional Capacity reduction $(R=-.622, P=.043)$, and in the HD 2 group, the reduction of mean MTR correlated to the reduction in Digit Technique Test performance $(R=-.667, P=.018)$. Both findings did not survive Bonferroni correction for multiple comparisons.

All volumetric values are shown in On-line Tables 3 and 4 . Significant volumetric decline of several VOIs was seen in the premanifest (caudate nucleus and putamen) and manifest groups (caudate nucleus, putamen, thalamus, white matter, and whole brain).

\section{DISCUSSION}

The main finding of this study is that MTI parameters do not change during a 2-year follow-up period in manifest HD. The expected decline in structural integrity in this group could not be detected with MTI. However, there was an interesting finding in the premanifest far from expected disease onset group - namely, an increase in the MTR value in the putamen, which possibly can be interpreted as a form of aberrant development or compensatory mechanism. There was no relationship of changing MTR parameters to increasing clinical symptoms in any of the groups. Clinical progression was evident in the premanifest close to disease onset group and the manifest HD groups.

Previous cross-sectional observations with MTI in HD showed promising results for both mean MTR and MTR histogram peak height for these measures to serve as a disease-monitoring biomarker. ${ }^{6,7,17}$ Mean MTR was found to be lower in several brain regions in manifest $\mathrm{HD}^{6}$ or in a combined cohort of premanifest and manifest $\mathrm{HD},{ }^{7}$ compared with controls. Also MTR peak height in several regions was found to be lower in manifest $\mathrm{HD},{ }^{6}$ and though there was no group difference, the MTR peak height related to subtle motor abnormalities and higher cytosine/adenosine-guanine repeat length in a premanifest HD cohort. ${ }^{17}$ To date, no longitudinal MTI studies in HD are available, to our knowledge. In fact, there is relatively little longitudinal research on MTI values available at all. Only in multiple sclerosis are optic neuritis and systemic lupus erythematosus reports available, suggesting a good potential for MTI to examine both gray and white matter for lesion evolution. ${ }^{26-29}$ In normal aging, Ge et al $(2002)^{30}$ described, via a histogram analysis, reduction of MTR values after 40 years of age and significant group differences after 50 years of age. The groups in our study spanned exactly this age range. We, therefore, calibrated our MTR values of the HD groups to the control values.

The goal of this study was to examine the potential of MTI to detect loss of structural integrity in HD to ultimately serve as an outcome measure in future therapeutic trails, as was the overall main goal of the TRACK-HD study. ${ }^{10}$ This current study and the recently published 24-month analysis of the main TRACK-HD study demonstrate that volumetric MR imaging measures are sensitive for detecting change during a 2-year period. ${ }^{31}$ In our MTI study, no statistically strong changes were seen in any of the regions. Three regions did show longitudinal reduction, namely the cortical gray matter in premanifest close to disease onset and the amygdala in HD 1 and 2; however, these findings did not survive correction for multiple comparison and thus should be interpreted with extreme caution. These regions are shown to be affected at these disease stages ${ }^{10,11}$; however, other regions such as the caudate nucleus or putamen or pallidum are known to be more severely affected in terms of atrophy. The MTR histogram peak height was normalized for volume, and mean MTR is nondependent on atrophy; only the number of voxels from which this mean is calculated could have an influence. The measures used are therefore relatively uninfluenced by atrophy. We must conclude from our study, on the basis of the group comparisons of MTI parameters, group comparisons of volumes, and the lack of correlation of MTI to clinical measures, that MTI is inferior to volumetric measures as a biomarker to detect longitudinal change in a 2-year follow-up. Clinical trials from a practical point of view will not last longer than 2 years; hence, a longer follow-up period may be of interest scientifically, but the impact on biomarker research in HD will be limited.

The interesting finding in this study in premanifest far from disease onset is that an increase in MTI values was observed. This pattern was seen in 5 subcortical gray matter structures, with the finding of increased MTR histogram peak height in the putamen, one of the most heavily involved structures in $\mathrm{HD}$, surviving the stringent correction for multiple comparisons. The explanation of this increase could be sought in an earlier postulated theory by Paulsen et al. ${ }^{32}$ They gave 2 possible explanations when their group detected increased cortical volume in premanifest HD. These explanations were either a predegenerative process, such as swelling of tissue, or alternatively increased cortical volume as a reflection of aberrant brain development or maturation. ${ }^{32}$

Furthermore, evidence from fMRI studies indicated another possible explanation. Two reports exist on increased activation in premanifest $\mathrm{HD}$, which is thought to represent cortical recruitment as a compensatory strategy; this was specifically true for premanifest HD far from expected onset and not in the close to onset group. ${ }^{33,34}$ In our view, any of these proposed explanations could be true; however, we should be extremely cautious not to overly interpret the findings because the correction for multiple comparisons reduced the number and strength of the findings. This finding could, however, lead to broader examination of MTI 
and other MR imaging measures in the premanifest far from disease onset group because this potentially can lead to better understanding of the neuropathology in HD.

One of the limitations of our study was that not all participants were retained for the follow-up period. However, those lost to follow-up were evenly divided over the groups; hence, we do not believe this ultimately influenced our results. Furthermore, the group sizes were relatively small, possibly suggesting that the study could be underpowered; however, combining the groups did not result in any additional findings. On the other hand, aggregated groups could be too heterogeneous to yield additional significant findings.

\section{CONCLUSIONS}

We believe that MTI-derived measures are not suitable for disease monitoring in HD because there was no significant decrease in structural integrity in any of the groups during 2 years and there was no significant relation to clinical measures. The finding of increased MTI measures in the premanifest far from disease onset group could relate to a predegenerative process, compensatory mechanisms, or aberrant development but should be interpreted with caution until confirmation of these findings in future studies is made.

\section{ACKNOWLEDGMENTS}

We thank B. Mertens from the Department of Medical Statistics for his input in the statistical analysis and Rachael Scahill for her input in the volumetric analysis. We also thank all TRACK-HD participants and investigators for their effort in conducting this study.

Disclosures: Ralf Reilmann—UNRELATED: Consultancy: Novartis, Lundbeck, CDHI, Siena Biotech, Ipsen, ISIS, Grants/Grants Pending: CHDI Foundation. Julie StoutUNRELATED: Consultancy: Prana Biotech, University of Michigan, Comments: In both cases, the consultant arrangements took place after the data for the study were collected and are not related to the results of the current study, Grants/ Grants Pending: Cure for Huntington's Disease Initiative, ${ }^{*}$ CHDI Foundation Inc.* *Money paid to the institution.

\section{REFERENCES}

1. Dehmeshki J, Chard DT, Leary SM, et al. The normal appearing grey matter in primary progressive multiple sclerosis: a magnetisation transfer imaging study. J Neurol 2003;250:67-74

2. Filippi M, Rocca MA. Magnetization transfer magnetic resonance imaging of the brain, spinal cord, and optic nerve. Neurotherapeutics 2007;4:401-13

3. McGowan JC. The physical basis of magnetization transfer imaging. Neurology 1999;53(5 suppl 3):S3-7

4. Ridha BH, Tozer DJ, Symms MR, et al. Quantitative magnetization transfer imaging in Alzheimer disease. Radiology 2007;244: 832-37

5. Eckert T, Sailer M, Kaufmann J, et al. Differentiation of idiopathic Parkinson's disease, multiple system atrophy, progressive supranuclear palsy, and healthy controls using magnetization transfer imaging. Neuroimage 2004;21:229-35

6. van den Bogaard SJ, Dumas EM, Milles J, et al. Magnetization transfer imaging in premanifest and manifest Huntington disease. AJNR Am J Neuroradiol 2012;33:884-89

7. Ginestroni A, Battaglini M, Diciotti S, et al. Magnetization transfer MR imaging demonstrates degeneration of the subcortical and cor- tical gray matter in Huntington disease. AJNR Am J Neuroradiol 2010;31:1807-12

8. A novel gene containing a trinucleotide repeat that is expanded and unstable on Huntington's disease chromosomes: the Huntington's Disease Collaborative Research Group. Cell 1993;72:971-83

9. Langbehn DR, Brinkman RR, Falush D, et al. A new model for prediction of the age of onset and penetrance for Huntington's disease based on CAG length. Clin Genet 2004;65:267-77

10. Tabrizi SJ, Langbehn DR, Leavitt BR, et al. Biological and clinical manifestations of Huntington's disease in the longitudinal TRACK-HD study: cross-sectional analysis of baseline data. Lancet Neurol 2009;8:791-801

11. van den Bogaard SJ, Dumas EM, Acharya TP, et al. Early atrophy of pallidum and accumbens nucleus in Huntington's disease. J Neurol 2011;258:412-20

12. Dumas EM, van den Bogaard SJ, Ruber ME, et al. Early changes in white matter pathways of the sensorimotor cortex in premanifest Huntington's disease. Hum Brain Mapp 2012;33:203-12

13. Rosas HD, Tuch DS, Hevelone ND, et al. Diffusion tensor imaging in presymptomatic and early Huntington's disease: selective white matter pathology and its relationship to clinical measures. Mov Disord 2006;21:1317-25

14. Rosas HD, Hevelone ND, Zaleta AK, et al. Regional cortical thinning in preclinical Huntington disease and its relationship to cognition. Neurology 2005;65:745-47

15. Henley SM, Frost C, Macmanus DG, et al. Increased rate of wholebrain atrophy over 6 months in early Huntington disease. Neurology 2006;67:694-96

16. Mascalchi M, Lolli F, Della NR, et al. Huntington disease: volumetric, diffusion-weighted, and magnetization transfer MR imaging of brain. Radiology 2004;232:867-73

17. Jurgens CK, Bos R, Luyendijk J, et al. Magnetization transfer imaging in 'premanifest' Huntington's disease. J Neurol 2010;257: 426-32

18. van Waesberghe JH, Kamphorst W, De Groot CJ, et al. Axonal loss in multiple sclerosis lesions: magnetic resonance imaging insights into substrates of disability. Ann Neurol 1999;46:747-54

19. Filippi M, Rocca MA, Comi G. The use of quantitative magneticresonance-based techniques to monitor the evolution of multiple sclerosis. Lancet Neurol 2003;2:337-46

20. Tabrizi SJ, Scahill RI, Durr A, et al. Biological and clinical changes in premanifest and early stage Huntington's disease in the TRACK-HD study: the 12-month longitudinal analysis. Lancet Neurol 2011;10:31-42

21. Penney JB Jr, Vonsattel JP, MacDonald ME, et al. CAG repeat number governs the development rate of pathology in Huntington's disease. Ann Neurol 1997;41:689-92

22. Zhang Y, Brady M, Smith S. Segmentation of brain MR images through a hidden Markov random field model and the expectation-maximization algorithm. IEEE Trans Med Imaging 2001;20: 45-57

23. Patenaude B, Smith SM, Kennedy DN, et al. A Bayesian model of shape and appearance for subcortical brain segmentation. Neuroimage 2011;56:907-22

24. Smith SM, Jenkinson M, Woolrich MW, et al. Advances in functional and structural MR image analysis and implementation as FSL. Neuroimage 2004;23(suppl 1):S208-19

25. Smith SM, Zhang Y, Jenkinson M, et al. Accurate, robust, and automated longitudinal and cross-sectional brain change analysis. $\mathrm{Neu}$ roimage 2002;17:479-89

26. Melzi L, Rocca MA, Marzoli SB, et al. A longitudinal conventional and magnetization transfer magnetic resonance imaging study of optic neuritis. Mult Scler 2007;13:265-68

27. Mesaros S, Rocca M, Sormani M, et al. Bimonthly assessment of magnetization transfer magnetic resonance imaging parameters in multiple sclerosis: a 14-month, multicentre, follow-up study. Mult Scler 2010;16:325-31 
28. Filippi M, Inglese M, Rovaris M, et al. Magnetization transfer imaging to monitor the evolution of MS: a 1-year follow-up study. Neurology 2000;55:940-46

29. Emmer BJ, Steens SC, Steup-Beekman GM, et al. Detection of change in CNS involvement in neuropsychiatric SLE: a magnetization transfer study. J Magn Reson Imaging 2006;24:812-16

30. Ge Y, Grossman RI, Babb JS, et al. Age-related total gray matter and white matter changes in normal adult brain. Part II. Quantitative magnetization transfer ratio histogram analysis. AJNR Am J Neuroradiol 2002;23:1334-41

31. Tabrizi SJ, Reilmann R, Roos RA, et al. Potential endpoints for clin- ical trials in premanifest and early Huntington's disease in the TRACK-HD study: analysis of $\mathbf{2 4}$ month observational data. Lancet Neurol 2012;11:42-53

32. Paulsen JS, Magnotta VA, Mikos AE, et al. Brain structure in preclinical Huntington's disease. Biol Psychiatry 2006;59:57-63

33. Zimbelman JL, Paulsen JS, Mikos A, et al. fMRI detection of early neural dysfunction in preclinical Huntington's disease. J Int Neuropsychol Soc 2007; 13:758-69

34. Paulsen JS, Zimbelman JL, Hinton SC, et al. fMRI biomarker of early neuronal dysfunction in presymptomatic Huntington's Disease. AJNR Am J Neuroradiol 2004;25:1715-21 